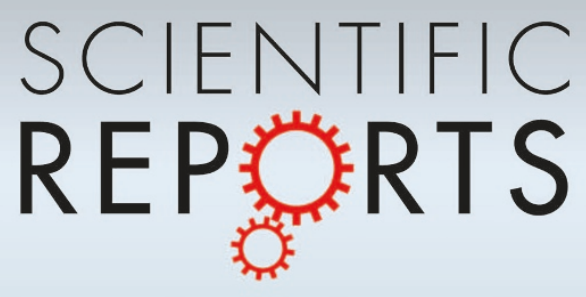

OPEN

SUBJECT AREAS:

QUANTUM

INFORMATION

QUBITS

THEORETICAL PHYSICS

SUPERCONDUCTING DEVICES

Received

3 October 2012

Accepted

4 March 2013

Published

26 March 2013

Correspondence and requests for materials should be addressed to D.V.A. (dmitri.averin@ stonybrook.edu)

\section{Decoherence induced deformation of the ground state in adiabatic quantum} computation

\author{
Qiang Deng' ', Dmitri V. Averin' ', Mohammad H. Amin ${ }^{2,3}$ \& Peter Smith 2,3
}

'Department of Physics and Astronomy, Stony Brook University, SUNY, Stony Brook, NY 1 1794-3800, '2D-Wave Systems Inc., 100-440 1 Still Creek Drive, Burnaby, B.C., Canada V5C 6G9, '3 Department of Physics, Simon Fraser University, Burnaby, British Columbia, Canada V5A 1S6.

Despite more than a decade of research on adiabatic quantum computation (AQC), its decoherence properties are still poorly understood. Many theoretical works have suggested that AQC is more robust against decoherence, but a quantitative relation between its performance and the qubits' coherence properties, such as decoherence time, is still lacking. While the thermal excitations are known to be important sources of errors, they are predominantly dependent on temperature but rather insensitive to the qubits' coherence. Less understood is the role of virtual excitations, which can also reduce the ground state probability even at zero temperature. Here, we introduce normalized ground state fidelity as a measure of the decoherence-induced deformation of the ground state due to virtual transitions. We calculate the normalized fidelity perturbatively at finite temperatures and discuss its relation to the qubits' relaxation and dephasing times, as well as its projected scaling properties.

diabatic quantum computation $(\mathrm{AQC})^{1,2}$, either in its universal form ${ }^{3,4}$, or in the form of adiabatic quantum optimization ${ }^{5,6}$, or quantum simulations ${ }^{7}$, presents a viable alternative to gate-model quantum computation (GMQC). Although a part of the original motivation for introduction of the AQC ${ }^{2}$ was the promise of the increased stability against decoherence due to the energy gap between the ground and excited states, the question of the role of decoherence in AQC remains an open one. This uncertainty makes it important to quantify more precisely the decoherence properties of AQC. A crucial step towards this would be to define a quantitative characteristic of the decoherence strength in AQC, that plays a role similar to the decoherence time for GMQC. However, in the case of AQC, decoherence has qualitatively different, static effect on the qubits, not limiting the operation time of an algorithm ${ }^{8}$.

In AQC, adiabatic evolution of the ground state of a qubit system realizes the solution of a computational problem represented by an appropriately designed Hamiltonian, which is typically written as

$$
H_{S}=A(s) H_{D}+B(s) H_{P},
$$

where $s=t / t_{f}$ with $t_{f}$ being the total evolution time. At $s=0$, one has $A(0)=1, B(0)=0$, and the system is initialized in the ground state of the initial (driver) Hamiltonian $H_{D}$, which usually consists of the uniform superposition of all computational basis states. The energy scales $A(s)$ and $B(s)$ are then varied monotonically so that at $s=1, A(1)=0$ and $B(1)=1$. If the evolution is slow enough, an isolated qubit system stays in the ground state with high fidelity throughout the evolution, and at $s=1$ reaches the ground state of the final (problem) Hamiltonian $H_{P}$, which provides a solution to a computational problem.

If the qubit system is weakly coupled to a dissipative environment, two effects are expected. First, the lowfrequency part of the environmental noise moves the system energy levels relative to each other. This results in a dephasing of the energy eigenstates that eventually suppresses all off-diagonal elements of the qubit density matrix in the energy basis. However, since the population of the ground state is the only important part of the computation and the relative phases of the energy eigenstates do not carry any information, this does not affect AQC. The second effect of the coupling to environment is that it induces thermal transitions between the qubit energy levels pushing the qubit system towards thermal equilibrium at a temperature $T$. In the limit of large $t_{f}$, the instantaneous probability to be in the ground state asymptotically approaches the Boltzmann distribution, and so the qubit system loses some of the ground state probability due to thermal occupation of the excited states. Such a thermal loss of probability can be compensated by multiple iterations of an AQC algorithm as long as it does not 
scale exponentially with the size of the system, i.e., as long as the number of excited states within roughly the energy $k_{B} T$ above the ground state does not grow exponentially.

The preceding arguments provide an intuitive explanation for the predicted robustness of AQC against local environmental noise in the limit of weak coupling ${ }^{8-15}$. When the strength of the coupling to the environment is increased without changing either the Hamiltonian or the temperature, the qubit system's Boltzmann distribution is still not directly affected. However, it is known that the decoherence time of the qubits decreases with increased coupling, and strong coupling to the environment eventually makes the qubits completely incoherent, rendering them useless for quantum computation. In GMQC, qubit decoherence leads to computation errors which, without error correction, completely destroy the computation process. This is why the qubits' quality factor, which is the ratio of the decoherence time and the gate operation time, provides a good measure of the qubit performance in GMQC. It is, however, unclear how an increase in coupling to the environment, or equivalently decrease in qubit quality factor, affects AQC.

In this paper, we look closely at what happens to the eigenstates of the qubit system in AQC when coupling to the environment is nonnegligible but still small enough to allow perturbation expansion. We introduce the normalized ground state fidelity, defined as the distance between the open and closed system reduced density matrices normalized to the Boltzmann ground state probability, as a quantitative measure of decoherence-induced deformation of the ground state in AQC, analogous to the decoherence time for GMQC. We calculate the fidelity perturbatively and express it through the same environmental noise correlators that determine the decoherence times in GMQC. Such an equilibrium calculation of the normalized ground state fidelity is accurate in the long $t_{f}$ limit, but becomes approximate when the evolution is too fast for the system to reach local equilibrium. However, the deviation from the equilibrium distribution is largest when the rate of relaxation between the eigenstates becomes extremely small (e.g., near the end of the evolution in adiabatic quantum optimization). As we shall see, the normalized ground state fidelity is closely related to the relaxation rate and becomes close to 1 , independent of the detailed probability distribution, when the relaxation is very slow. Therefore, our calculation of the normalized fidelity can provide a good approximation in all regions as long as the evolution time is not too short.

\section{Results}

Normalized ground state fidelity-definition. We first provide a definition for the normalized ground state fidelity based on the notion of fidelity between two density matrices. To ensure consistent notation throughout this paper, symbols with (without) " " denote quantities related to the coupled (uncoupled) qubit system and environment. We use letters $m, n$ to enumerate the eigenstates and eigenvalues of the qubit system (e.g., $|n\rangle, E_{n}$ ), letters $v, \mu$ to enumerate the eigenstates and eigenvalues of the environmental degrees of freedom, and letters $a, b$ to enumerate the eigenstates and eigenvalues of the total system (qubits + environment). The total Hamiltonian is $\tilde{H}=H_{S}+H_{B}+H_{I}$, where $H_{B}$ and $H_{I}$ are the environment and interaction Hamiltonians, respectively. In the absence of coupling, $H_{I}=0$, and the eigenstates of the total system are $|a\rangle=|n\rangle \otimes|v\rangle$ with eigenvalues $E_{a}=E_{n}+E_{v}$. When $H_{I} \neq 0$, the new eigenstates are $|\tilde{a}\rangle$, which typically are entangled superpositions of the unperturbed states $|a\rangle$. For weak coupling, $|\tilde{a}\rangle$ is very close to $|a\rangle$ and the effect of the environment is thermalization of the qubit system. Once the environment is averaged out, the equilibrium of the total system gives the Boltzmann distribution for the qubits:

$$
P_{n}=\sum_{v} \frac{e^{-\left(E_{n}+E_{v}\right) / T}}{Z_{S} Z_{B}}=\frac{e^{-E_{n} / T}}{Z_{S}},
$$

where $Z_{S}=\sum_{n} e^{-E_{n} / T}$ and $Z_{B}=\sum_{v} e^{-E_{v} / T}$ are the partition functions of the qubit system and the environment.

As the coupling strength increases, the deviation of $|\tilde{a}\rangle$ from $|a\rangle$ grows. In equilibrium, the density matrix of the total system still has the Boltzmann form $\tilde{\rho}_{S B}=\sum_{a} \tilde{P}_{a}|\tilde{a}\rangle\langle\tilde{a}|$, where $\tilde{P}_{a}=e^{-\tilde{E}_{a} / T} / \tilde{Z}_{S B}$, with $\tilde{Z}_{S B}=\sum e^{-\tilde{E}_{a} / T}$ being the partition function of the total system. However, the reduced density matrix $\tilde{\rho}_{S}=\operatorname{Tr}_{B}\left[\tilde{\rho}_{S B}\right]$ of the qubit system alone is no longer given by the Boltzmann distribution. The deviation from the Boltzmann form provides a good qualitative measure of how strongly the eigenstates $|\tilde{a}\rangle$ are deformed in comparison to the unperturbed states.

To quantify the loss of fidelity of the ground state due to such deformation of the energy eigenstates of the uncoupled system, it is convenient first to separate this effect from the loss of fidelity due to thermal excitations. This can be done by using normalized ground state fidelity, which we define as the Uhlmann fidelity ${ }^{16}$ between the reduced density matrix $\tilde{\rho}_{S}$ and the "ideal" ground state density matrix $\rho_{0}=|0\rangle\langle 0|$, normalized to the Boltzmann ground state probability $P_{0}$ :

$$
F=P_{0}^{-1 / 2} \operatorname{Tr} \sqrt{\sqrt{\rho_{0}} \tilde{\rho}_{S} \sqrt{\rho_{0}}}=\sqrt{\tilde{P}_{0} / P_{0}}
$$

where $\tilde{P}_{0}=\left\langle 0\left|\tilde{\rho}_{S}\right| 0\right\rangle$ is the equilibrium probability for the qubits to be in the ideal ground state when coupled to the environment. Normalization to the equilibrium Boltzmann ground state probability $P_{0}$ is natural in the context of this work, since in the calculations presented below we adopt the assumption that the qubitenvironment system maintains equilibrium throughout the AQC evolution. In the weak-coupling limit, no deformation of the eigenstates is expected. Then $\tilde{P}_{0}=P_{0}$, and Eq. (3) gives $F=1$. This shows that Eq. (3) indeed correctly separates the effect of the quantum deformation of the ground state, which can be viewed as the result of virtual transitions to the excited states, from the thermal loss of probability. Qualitatively, the effect of the virtual transitions, expressed in $F$, is different from that of the thermal transitions in two important aspects. First, it persists even at $T=0$, when all the thermal transitions are suppressed. Second, it depends on the strength of coupling to the environment (or decoherence time of the qubits), while thermal equilibrium probabilities only depend on the energy eigenvalues and temperature. Nevertheless, similarly to the thermal transitions, the virtual transitions reduce the occupation probability of the ground state by transferring it to other low energy states. In general, beyond the AQC, deviation of the occupation probabilities of an equilibrium quantum system from the Boltzmann distribution due to non-vanishing strength of coupling to environment has been studied before as introducing corrections to classical thermodynamics (see, e.g. Ref. 17-21, and references therein). In particular, deformation of the ground state by coupling to an environment, described in our case as a suppression of fidelity, is known to lead to several physical effects, e.g., suppression of the persistent current in normal-metal rings ${ }^{22}$ or violation of the fluctuation-dissipation theorem for the thermal conductance ${ }^{23}$.

Perturbative calculation of fidelity. We calculate the fidelity (3) perturbatively and relate it to measurable parameters of the qubit system and environment. As appropriate for AQC, we assume that the coupling $H_{I}$ is weak. This allows us to employ the perturbation theory in $H_{I}$ around the non-interacting state of the qubit system and the environment. To separate the effects of environment from other deviations from the perfect adiabatic evolution of an AQC algorithm, we consider the limit of slow evolution, when the rate of change of the control parameter of the Hamiltonian is small, e.g., smaller than the energy relaxation rate of the qubit system. In this case, non-adiabatic transitions out of the ground state can be neglected, and the occupation probabilities of the excited states (if non-vanishing) correspond to local equilibrium at each moment during the 
evolution. While this is obviously not the most general case of time evolution in AQC, which can be dominated by non-equilibrium effects, it covers the most important regime of ideal AQC evolution, and is appropriate for the situation of sufficiently slow evolution in the presence of small but finite interaction strength with environment we want to describe. The zeroth-order state of the perturbation theory in this regime is the state with both the uncoupled qubit system and environment in equilibrium at the same temperature $T$, i.e., the total density matrix of the system being the product of the density matrices $\rho_{S}=\sum_{n} P_{n}|n\rangle\langle n|$ and $\rho_{B}=\sum_{v} P_{v}|v\rangle\langle v|$, where $P_{n}$ and $P_{v}$ are the Boltzmann probabilities.

In general, the reduction of the ground state probability due to finite $H_{I}$ is caused by two effects. First is the change $\delta P_{0}$ in the equilibrium probability $P_{0}\left(\equiv P_{n=0}\right)$ as a result of renormalization of the energy eigenvalues (Lamb shifts) of the qubit system. Second effect, conceptually more important for this work, is the probability transfer into and out of the ground state due to renormalization of the qubit system wavefunctions. Explicitly, the probability $\tilde{P}_{0}$ that defines the normalized fidelity (3) can be expressed as

$$
\tilde{P}_{0} \equiv \tilde{P}_{n=0}=\operatorname{Tr}_{B, S}\left[|0\rangle\left\langle 0\left|\sum_{a} \tilde{P}_{a}\right| \tilde{a}\right\rangle\langle\tilde{a}|\right] .
$$

Introducing interaction-induced corrections to the equilibrium probabilities $\widetilde{P}_{a}=P_{a}+\delta P_{a}$, where $P_{a}=P_{n} P_{v}$, and wavefunctions: $|\tilde{a}(n, v)\rangle=|n\rangle \otimes|v\rangle+|\delta \tilde{a}(n, v)\rangle$, we can rewrite this expression to the lowest non-vanishing order in $H_{I}$ as

$$
\tilde{P}_{0}=\delta P_{0}+\sum_{n} P_{n} \operatorname{Tr}_{B, S}\left[|0\rangle\left\langle 0\left|\otimes \rho_{B} \cdot\right| \tilde{a}(n, v)\right\rangle\langle\tilde{a}(n, v)|\right] .
$$

Using the relation $|0\rangle\left\langle 0\left|=1-\sum_{m \neq 0}\right| m\right\rangle\langle m|$ to transform the $n=0$ term in Eq. (5) we obtain

$$
\tilde{P}_{0}=P_{0}+\delta P_{0}-\sum_{n \neq 0}\left(\Gamma_{0 n} P_{0}-\Gamma_{n 0} P_{n}\right),
$$

where

$$
\Gamma_{m n} \equiv\left\langle n\left|\operatorname{Tr}_{B}\left[|\delta \tilde{a}(m, v)\rangle\langle\delta \tilde{a}(m, v)| \rho_{B}\right]\right| n\right\rangle .
$$

The terms proportional to $\Gamma$ in Eq. (6) describe the reduction of the ground state probability as a result of virtual transitions between the ground and excited state due to the interaction with the environment.

Next, we calculate $\delta P_{0}$ and $\Gamma_{m n}$. Quite generally, the interaction Hamiltonian $H_{I}$ is

$$
H_{I}=\sum_{j, \alpha} q_{j}^{\alpha} \sigma_{j}^{\alpha}
$$

where $\sigma_{j}^{\alpha}$ are the Pauli matrices for the $j$ th qubit, $\alpha=x, y, z$, and $q_{j}^{\alpha}$ are the corresponding operators of the noise generated by the environment. As usual, the averages of the noise operators vanish, $\left\langle q_{j}^{\alpha}\right\rangle=0$. Then, in the weak coupling regime, the effect of environment is fully characterized by the noise spectral densities:

$$
S_{j}^{\alpha}(\omega)=\int d t e^{i \omega t}\left\langle q_{j}^{\alpha}(t) q_{j}^{\alpha}(0)\right\rangle,
$$

where $\langle\ldots\rangle=\operatorname{Tr}_{B}\left\{\rho_{B} \ldots\right\}$ is the average over the environmental degrees of freedom. For simplicity, we limit our discussion to the most typical case when the noises with different $\alpha$ or $j$ are uncorrelated. It is shown in the supplementary information (SI) that the perturbation expansion in $H_{I}$ in this situation gives

$$
\begin{aligned}
& \delta P_{0}=-\beta P_{0} \sum_{j, \alpha, n, m}\left(P_{n}-\delta_{n 0}\right)\left|\sigma_{j, n m}^{\alpha}\right|^{2} \int \frac{d \omega}{2 \pi} \frac{S_{j}^{\alpha}(\omega)}{\omega_{m n}+\omega}, \\
& \Gamma_{m n}=\sum_{j, \alpha}\left|\sigma_{j, n m}^{\alpha}\right|^{2} \int \frac{d \omega}{2 \pi} \frac{S_{j}^{\alpha}(\omega)}{\left(\omega_{n m}+\omega\right)^{2}},
\end{aligned}
$$

where $\sigma_{j, n m}^{\alpha} \equiv\left\langle n\left|\sigma_{j}^{\alpha}\right| m\right\rangle$ and $\omega_{n m} \equiv E_{n}-E_{m}$. Substituting (9) into (6) and then into (3), we obtain

$$
\begin{aligned}
F= & 1-\beta \sum_{j, \alpha, n, m}\left|\sigma_{j, n m}^{\alpha}\right|^{2} \int \frac{d \omega}{4 \pi} \frac{S_{j}^{\alpha}(\omega)\left(P_{n}-\delta_{n 0}\right)}{\omega_{m n}+\omega} \\
& -\sum_{j, \alpha, n>0}\left|\sigma_{j, n 0}^{\alpha}\right|^{2} \int \frac{d \omega}{4 \pi} \frac{S_{j}^{\alpha}(\omega)-\left(P_{n} / P_{0}\right) S_{j}^{\alpha}(-\omega)}{\left(\omega_{n 0}+\omega\right)^{2}} .
\end{aligned}
$$

Equation (10) is our main result. The normalized fidelity is welldefined at $T=0$, when all thermal excitations are suppressed, i.e., $P_{n}=0$ for $n>0$ and $S_{j}^{\alpha}(\omega) \equiv 0$ at $\omega<0$. Hence, the values of $\omega$ around $-\omega_{m 0}$, when the denominator in (10) vanishes, do not contribute to the integral. When $T \neq 0$, the divergences that appear at $\omega$ $=-\omega_{m 0}$ reflect the fact that environment can also create real thermal excitations of the qubit system. However, the detailed balance relation, $S_{j}^{\alpha}(-\omega)=e^{-\beta \omega} S_{j}^{\alpha}(\omega)$, ensures that these divergences cancel each other out and Eq. (10) is well-defined also at $T \neq 0$ (see the SI).

Normalized fidelity for single qubit. Equation (10) is now applied to specific problems. The first example we consider is a typical individual qubit with the Hamiltonian

$$
H_{S}=-\left[\varepsilon \sigma^{z}+\Delta \sigma^{x}\right] / 2
$$

coupled as in Eq. (7), but only through $\sigma^{z}$, to the environmental noise with spectral density $S(\omega)$ (8). In the usual weak-coupling approximation [see, e.g., Ref. 24], the qubit decoherence time $T_{2}^{*}$ is given by

$$
\frac{1}{T_{2}^{*}}=\frac{1}{2 T_{1}}+\frac{1}{T_{\varphi}},
$$

where $T_{1}$ and $T_{\varphi}$ are the relaxation and pure dephasing times, given by

$$
\begin{gathered}
T_{1}^{-1}=\left(\Delta^{2} / \Omega^{2}\right)[S(\Omega)+S(-\Omega)], \\
T_{\varphi}^{-1}=\left(\epsilon^{2} / \Omega^{2}\right) S(0),
\end{gathered}
$$

with $\Omega=\sqrt{\Delta^{2}+\epsilon^{2}}$. The standard expressions for the eigenstates of the Hamiltonian (11) reduce Eq. (10) for the normalized fidelity to $F=1-\Delta^{2} K / 2 \Omega^{2}$, where

$$
K=\int \frac{d \omega S(\omega-\Omega)}{2 \pi \omega}\left\{\frac{1-e^{-\omega / T}}{\omega}-\frac{e^{-\Omega / T}+e^{-\omega / T}}{T\left(e^{-\Omega / T}+1\right)}\right\} .
$$

We see that the same noise spectral density that defines the relaxation and dephasing rates (13) and (14) of the qubits in the GMQC determines the reduction of the ground-state normalized fidelity in AQC. In this respect, the main difference between the reduction of normalized fidelity and the real-time relaxation and dephasing is that even in the lowest-order perturbation theory, the normalized fidelity is reduced by the whole spectrum of environmental excitations, and not just by limited spectral groups resonant with the qubit energy differences or the low-frequency excitations, as in Eqs. (13) and (14).

To strengthen this comparison, we consider an Ohmic bath characterized by the noise

$$
S(\omega)=\eta \omega /\left(1-e^{-\omega / T}\right)
$$

where, $\eta$ is a dimensionless coefficient and $\omega_{c}$ is the cutoff frequency. In this case, the relaxation time is $T_{1}^{-1}=\eta\left(\Delta^{2} / \Omega\right) \operatorname{coth}(\Omega / 2 T)$ and the normalized fidelity is expressed as

$$
F=1-\frac{k}{Q}, \quad k \equiv \frac{K}{2 \eta} \tanh \frac{\Omega}{2 T},
$$

where $Q=T_{1} \Omega$ is the qubit quality factor due to relaxation. Equation (15) gives the following expressions for the factor $k$ at low and high temperatures: 


$$
k=\frac{1}{4 \pi}\left\{\begin{array}{ll}
\ln \left(\omega_{c} / \Omega\right)-1+\pi^{2} T^{2} / 3 \Omega^{2}, & T \ll \Omega, \\
(\Omega / T)^{2} \ln \left(\omega_{c} / T\right), & T \gg \Omega .
\end{array}\right\}
$$

Equation (17) relates the normalized ground state fidelity to the qubit quality factor, $Q$, as calculated due to relaxation only. This shows that the normalized fidelity can be related more closely to the relaxation $\left(T_{1}\right)$ and not dephasing $\left(T_{\varphi}\right)$ processes. Adding a $1 / f$ low-frequency noise of a realistic magnitude does not change this conclusion (as discussed in more details in the numerical examples below). As expected, a larger $Q$ leads to a better $F$. Figure 1 shows the factor $k$ in Eq. (17) as a function of temperature for different cut-off frequencies $\omega_{c}$. It exhibits the non-monotonic $T$-dependence, and only weak, logarithmic, dependence on $\omega_{c}$, which allows one to estimate $F$ without precisely specifying $\omega_{c}$. The factor $k$ is maximal around $k_{\max } \simeq 0.5$ at $T \simeq 0.5 \Omega$, which leads to a minimum normalized fidelity $F \simeq 1-(2 Q)^{-1}$. Notice that even a qubit quality factor as low as $Q=10$, which is practically useless for GMQC, leads to $F>95 \%$.

Normalized fidelity for multi-qubit systems. We now consider multi-qubit systems, starting with a system of $N$ uncoupled qubits. In this case, the trace in the definition of normalized fidelity (3) can be taken independently over separate qubits, so that the total $F$ is the product of fidelities $F_{j}, j=1, \ldots, N$ of the individual qubits: $F=\Pi_{j} F_{j}$. For instance, a typical starting point of $A Q C$ algorithms is to initialize the system in the ground state of the Hamiltonian $H_{D}(20)$. Then, the state of all qubits is the same and can be characterized by the same normalized fidelity (17). Then,

$$
F=\left.(1-k / Q)^{N}\right|_{Q \gg k} \simeq e^{-k N / Q} .
$$

For independent qubits, $F$ scales exponentially with $N$ as a result of the exponential scaling of the probability for all qubits to remain in their corresponding ground states. Since $Q$ is inversely proportional to the noise strength $\eta$, by decreasing the noise by a factors of, e.g., 10 , one can achieve the same $F$ with 10 times more qubits.

Next, we focus on how the normalized ground state fidelity behaves in practical AQC systems. We use as an example the $D$ Wave One quantum annealing processor, as the one installed at the University of Southern California (see Ref. 6). The Hamiltonian implemented by the processor has the form of Eq. (1), with

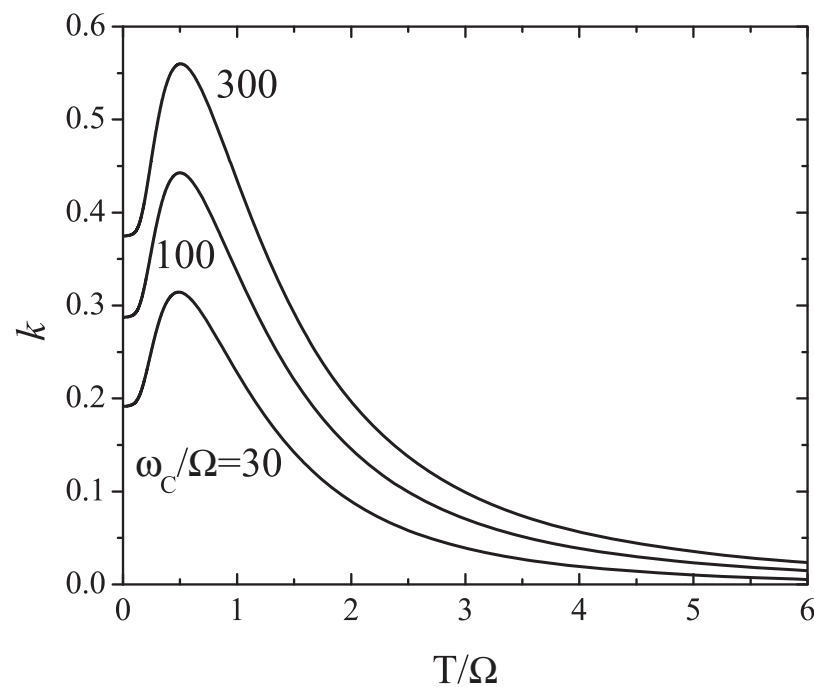

Figure $1 \mid$ The temperature-dependent factor $k$ in the expression (17) for the normalized ground state fidelity of an individual qubit in the presence of Ohmic environment with cut-off frequency $\omega_{c}$.

$$
H_{D}=-\sum_{i=1}^{N} \sigma_{i}^{x}, \quad H_{P}=\sum_{i=1}^{N} h_{i} \sigma_{i}^{z}+\sum_{i, j=1}^{N} J_{i j} \sigma_{i}^{z} \sigma_{j}^{z},
$$

where $h_{i}$ and $J_{i j}$ are tunable dimensionless bias and coupling coefficients. The parameters $A(s)$ and $B(s)$ for this processor are plotted in Fig. 2b. We calculate $F(s)$ for a ferromagnetic chain (illustrated in Fig. 2a) with $h_{i}=0$ and $J_{i, i+1}=-1$, otherwise known as a quantum Ising model in a transverse field. Here, the length of the chain is varied from $N=2$ to 16 . Although this model is exactly solvable (see, e.g., Ref. 27 and references therein), $F$ cannot be calculated exactly for practical noise models in which the coupling to environment is dominated by the $\sigma_{j}^{z}$ terms. Hence, we calculate the normalized fidelity numerically. In the limit $N \rightarrow \infty$, the model is known to have a quantum critical point at $s_{c}$ where $A\left(s_{c}\right)=B\left(s_{c}\right)$. At this point, the chain goes through a quantum phase transition between quantum paramagnetic and ferromagnetic phases. In the ferromagnetic phase, the ground state is doubly degenerate with respect to simultaneous change of signs of all $\sigma_{i}^{z}$ terms. Figure $2 \mathrm{c}$ plots several of the lowest energy levels of a 10-qubit chain relative to the ground state energy $E_{0}$. In the thermodynamic limit $(N \rightarrow \infty)$, the appearance of the doubly-degenerate ground state and the minimum in the energy gap between the ground and the second excited states happen at the quantum critical point. For the 10-qubit chain of Fig. 2, however, these happen at slightly different points than the one defined by $A\left(s_{c}\right)=B\left(s_{c}\right)$.

To calculate $F(s)$ for this system, we use a realistic noise model relevant to the $D$-Wave qubits ${ }^{25}$. In this case, the dominant environmental coupling is to the magnetic flux noise, which couples directly to the qubit computational basis states represented by the $\sigma_{j}^{z}$ operators. The noise spectral density $S(\omega)$ was characterized in the earlier experiments, which were consistent with the noise being a combination of the $1 / f$ low-frequency noise and an Ohmic noise at high frequencies ${ }^{26}$. For calculations of $F$, we take $S(\omega)=\kappa(s)\left[S_{H F}(\omega)\right.$ $+S_{L F}(\omega)$ ], where $S_{H F}(\omega)$ is the Ohmic spectral density (16) and $S_{L F}(\omega)=\gamma^{2} /|\omega|$. The coefficient $\kappa(s)=B(s) / B\left(s_{m}\right)$ appears because the strength of coupling to flux noise depends on the persistent current of the flux qubits which changes as a function of $s$ (see SI). Here, $s_{m}$ is the bias point at which the measurements of $\eta$ and $\gamma$ are performed. Based on the experimental data, we use $\eta=0.1, \gamma=$ $20 \mathrm{MHz}$ and $s_{m}=0.636$. We also assume $\omega_{c}=100 \mathrm{GHz}$ for the high-frequency cutoff and $\omega_{L}=1 \mathrm{MHz}$ for the low-frequency cutoff (based on a $t_{f} \sim 1 \mu$ s evolution time of an algorithm). We found that for these parameters, $F$ is dominantly determined by the high-frequency Ohmic noise and not by the $1 / f$ noise.

In principle, since the total number of energy levels grows exponentially with $N$, the time required for numerical calculation of $F$ also grows exponentially. Fortunately, the value of $F$ converges rapidly for a finite number of retained energy states. Here, we keep all energy levels for $N \leq 10$, and up to 2000 energy levels for larger chains. The normalized ground state fidelity $F(s)$ of the 10 -qubit chain is plotted as a function of $s$ in Fig. 2d. The fidelities of chains with other lengths (and also coupled systems other than chains) are qualitatively the same as the one plotted in Fig. 2d. It is clear from the figure that $F(s)$ is minimum close to the critical point $s_{c}$. Notice also that the fidelity approaches 1 as $s \rightarrow 1$, which is the result of $H_{P}$ commuting with $H_{I}$, with only $\sigma_{j}^{z}$ terms and negligible other types of coupling to environment. This again reflects the fact that $F$ depends rather on relaxation than dephasing.

Figure 3 shows the numerical results for the normalized ground state fidelity for $N$-qubit chains with $N=1$ to 16 at the critical point. For all chain lengths, $F\left(s_{c}\right)$ is better than $90 \%$. It should be emphasized that these are the minimum fidelities at the quantum critical point $s_{c}$. The normalized fidelity at all other points is larger, and near $s=1$, is very close to 1 as shown in Fig. 2d. We have also plotted in Fig. 3 the normalized ground state fidelity of $N$ uncoupled qubits 

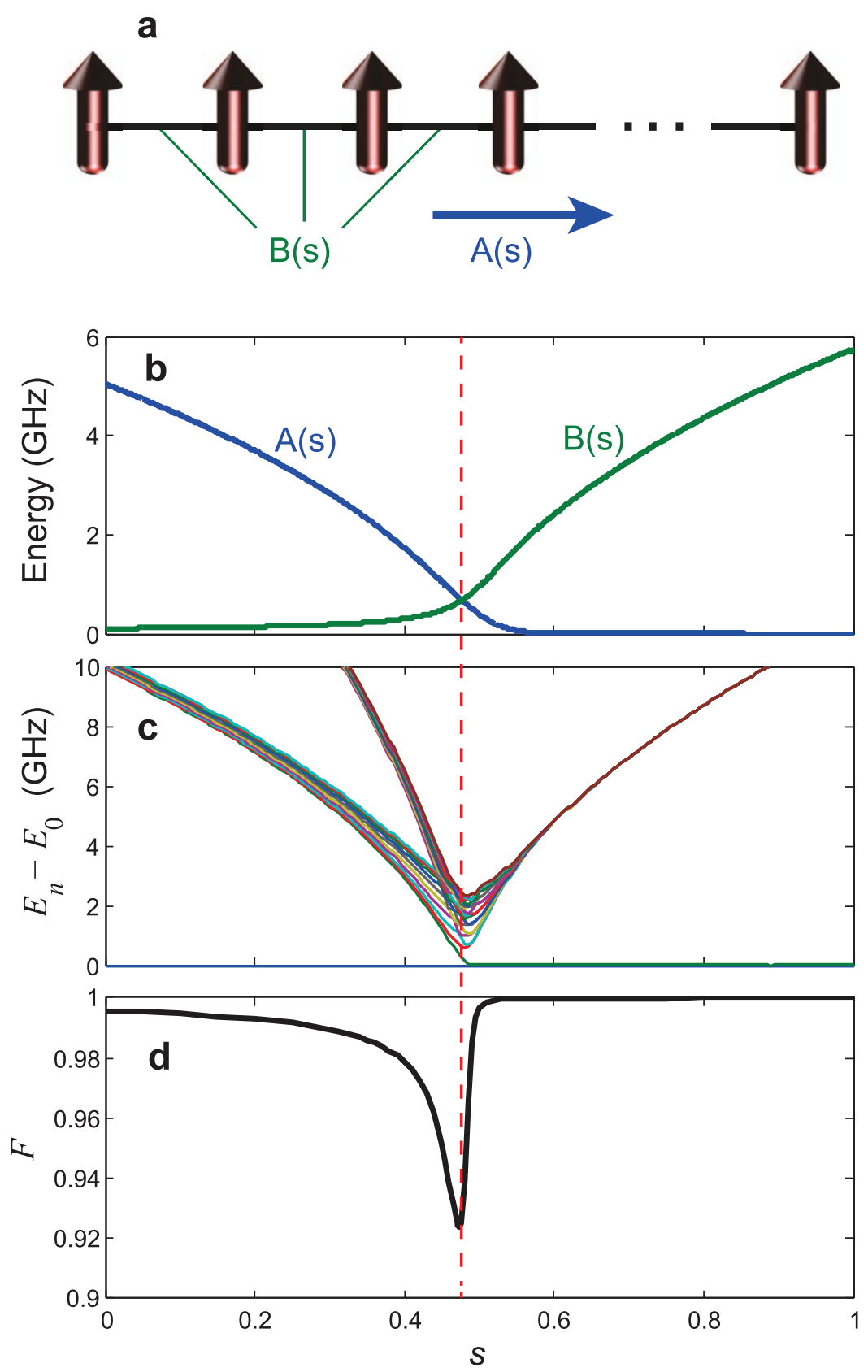

Figure $2 \mid$ (a) A ferromagnetic spin chain with transverse field and coupling energies given, respectively, by $A(s)$ and $B(s)$ in Eq. (1). (b) Energy scales $A(s)$ and $B(s)$ extracted from experimental parameters. (c) The lowest 20 energy levels, relative to the ground state, of a 10-qubit ferromagnetic chain with $J_{i j}=$ -1 , as a function of the normalized time $s$. (d) Normalized ground state fidelity of the 10-qubit chain of (c) at $T=20 \mathrm{mK}$. The vertical (red) dashed line marks the quantum critical point as defined by the condition $A\left(s_{c}\right)=B\left(s_{c}\right)$.

(with the same parameters) at different $N$ based on the exponential scaling of Eq. (19). The scaling and magnitude of $F$ at large $N$ is better for the ferromagnetic chain than for the uncoupled qubits. A plausible reason for this is that the spin-spin interaction introduces additional rigidity into the chain dynamics making it less susceptible to the environmental perturbations, and therefore increasing the fidelity. Unfortunately, it was not possible to pursue numerical calculations beyond 16 qubits, as direct perturbation approximation would break down when $F$ strongly deviates from unity. A naive exponential extrapolation of the data points to $N=128$ (representing the worse case) yields a lower bound of 0.47 for $F$, meaning that the eigenstates could retain their quantum properties without error correction for such a large-size system. As in uncoupled qubits, if one can reduce the noise by a large factor, the size of the chain can be increased by the same factor while keeping $F$ unchanged. In addition, other techniques such as dynamical decoupling ${ }^{28}$ or error correction $^{29}$ could be employed to enhance the normalized ground state fidelity at large scales.

\section{Discussion}

Finally, we discuss how the normalized ground state fidelity should affect the performance of AQC. First, we notice that the actual equilibrium ground state probability at point $s$ is $\tilde{P}_{0}(s)=P_{0}(s) F^{2}(s)$, where $P_{0}(s)$ is the Boltzmann probability. Therefore, a suppression of the normalized fidelity creates an extra reduction of the ground state probability on top of the thermal reduction. In universal $\mathrm{AQC}^{3,4}, F(1)$ directly affects the quality of the computation. Indeed, deviations of $F(1)$ from 1 mean that the statistics of measure- 


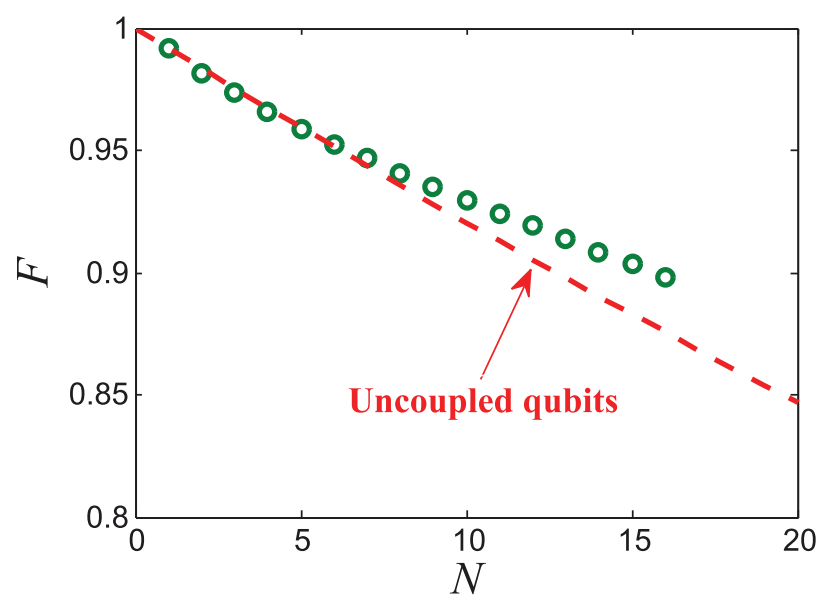

Figure $3 \mid$ Normalized ground state fidelity at the quantum critical point for ferromagnetic chains with $N=1$ to 16 , at $T=20 \mathrm{mK}$. Circles are the numerical results using (10). The red dashed line is the fidelity of uncoupled qubits from (19), with $k=0.32$ and $Q=38.4$.

ments done on the final state will be different from the one that corresponds to the ideal ground state. For instance, in the case of one qubit with $\epsilon=0$ and the Hamiltonian (11), measurement of $\sigma_{x}$ has a non-vanishing probability $1-F^{2}$ of producing the result $\sigma_{x}=$ -1 different from the ground state $\sigma_{x}=1$ even at temperatures $T \ll \Delta$. However, this effect is absent in the special case when the coupling to environment via $H_{I}$ commutes with the final Hamiltonian $H_{P}$, leading to $F=1$ at the end of evolution, as in the adiabatic quantum optimization discussed above and shown in Fig. 2d. In this case, a small fidelity in the middle of the evolution increases the loss of probability due to the thermal transitions, thereby decreasing the ground state probability even further. Therefore, the probability will be distributed among the low energy states even more than implied by the thermal equilibrium. Part of the probability can be regained later when the gap is larger and $F$ is closer to 1 . However, since the relaxation time becomes exponentially long near the end of evolution, the majority of the probability that is lost may not be gained back, thus leading to a smaller probability of success. This makes it important to maintain $F(s)$ close to unity throughout the evolution. We stress that most treatments of AQC based on the weak coupling master equation, e.g. Ref. 8,9,30, even with Lamb shift, do not take into account the effect of deformation of the eigenstates that is captured by our calculation of the normalized fidelity.

In summary, we have proposed using normalized ground state fidelity as a quantity for measuring the strength of decoherence effects in AQC. The fidelity plays a role similar to decoherence time in GMQC, but takes into account qualitatively different effects of environment on the ground state relevant to AQC. The fidelity is related to the relaxation processes and is relatively insensitive to the dephasing. Our numerical calculations indicate that a normalized fidelity close to unity can be achieved with a moderate qubit quality factor, even for large numbers of qubits. Normalized ground state fidelity should be a useful measure of the environment related quality of AQC systems in the context of further work on important topics in AQC such as quantum error correction or the threshold theorem.

1. Farhi, E. et al. A Quantum adiabatic evolution algorithm applied to random instances of an NP-complete problem. Science 292, 472 (2001).

2. Mizel, A., Mitchell, M. W. \& Cohen, M. L. Energy barrier to decoherence. Phys. Rev. A 63, 040302 (2001).

3. Aharonov, D. et al. Adiabatic quantum computation is equivalent to standard quantum computation. SIAM J. Comput. 37, 166 (2007).
4. Mizel, A., Lidar, D. A. \& Mitchell, M. Simple proof of equivalence between adiabatic quantum computation and the circuit model. Phys. Rev. Lett. 99, 070502 (2007).

5. Johnson, M. W. et al. Quantum annealing with manufactured spins. Nature 473, 194 (2011).

6. Bian, Z., Chudak, F., Macready, W. G., Clark, L., Gaitan F. Experimental determination of Ramsey numbers with quantum annealing. eprint arXiv:1201.1842.

7. Biamonte, J. D., Bergholm, V., Whitfield, J. D., Fitzsimons, J. \& Aspuru-Guzik, A. Adiabatic quantum simulators. AIP Advances 1, 022126 (2011).

8. Amin, M. H. S., Truncik, C. J. S. \& Averin, D. V. Role of single qubit decoherence time in adiabatic quantum computation. Phys. Rev. A 80, 022303 (2009).

9. Childs, A. M., Farhi, E. \& Preskill, J. Robustness of adiabatic quantum computation. Phys. Rev. A 65, 012322 (2001).

10. Roland, J. \& Cerf, N. J. Noise resistance of adiabatic quantum computation using random matrix theory. Phys. Rev. A 71, 032330 (2005).

11. Sarandy, M. S. \& Lidar, D. A. Adiabatic quantum computation in open systems. Phys. Rev. Lett. 95, 250503 (2005).

12. Tiersch, M. \& Schützhold, R. Non-Markovian decoherence in the adiabatic quantum search algorithm. Phys. Rev. A 75, 062313 (2007).

13. Amin, M. H. S., Love, P. J. \& Truncik, C. J. S. Themally assisted adiabatic quantum computation. Phys. Rev. Lett. 100, 060503 (2008).

14. Amin, M. H. S., Averin, D. V. \& Nesteroff, J. A. Decoherence in adiabatic quantum computation. Phys. Rev. A 79, 022107 (2009).

15. Lloyd, S. Robustness of adiabatic quantum computing. eprint arXiv:0805.2757.

16. Uhlmann, A. The transition probability in the state space of a *-algebra. Rep. Math. Phys. 9, 273 (1976).

17. Allahverdyan, A. E. \& Nieuwenhuizen, T. M. Extraction of work from a single thermal bath in the quantum regime. Phys. Rev. Lett. 85, 1799 (2000).

18. Nagaev, K. E. \& Büttiker, M. Ground-state energy fluctuations of a system coupled to a bath. Europhys. Lett. 58, 475 (2002).

19. Scully, M. O., Zubairy, M. S., Agarwal, G. S. \& Walther, H. Extracting work from a single heat bath via vanishing quantum coherence. Science 299, 862 (2003).

20. Zhang, W., Sun, C. P. \& Nori, F. Equivalence condition for the canonical and microcanonical ensembles in coupled spin systems. Phys. Rev. E 82, 041127 (2010).

21. Williams, N. S., Le Hur, K. \& Jordan, A. N. Effective thermodynamics of strongly coupled qubits. J. Phys. A 44, 385003 (2011).

22. Cedraschi, P., Ponomarenko, V. V. \& Büttiker, M. Zero-Point fluctuations and the quenching of the persistent current in normal metal rings. Phys. Rev. Lett. 84, 346 (2000).

23. Averin, D. V. \& Pekola, J. P. Violation of the fluctuation-dissipation theorem in time-dependent mesoscopic heat transport. Phys. Rev. Lett. 104, 220601 (2010).

24. Blum, K. Density matrix theory and applications (Plenum, New York, 1981).

25. Harris, R. et al. Experimental demonstration of a robust and scalable flux qubit. Phys. Rev. B 81, 134510 (2010).

26. Lanting, T. et al. Probing high-frequency noise with macroscopic resonant tunneling. Phys. Rev. B 83, 180502(R) (2011).

27. Dziarmaga, J. Dynamics of a quantum phase transition: exact solution of the quantum Ising model. Phys. Rev. Lett. 95, 245701 (2005).

28. Lidar, D. A. Towards fault tolerant adiabatic quantum computation. Phys. Rev. Lett. 100, 160506 (2008).

29. Jordan, S., Farhi, E. \& Shor, P. Error-correcting codes for adiabatic quantum computation. Phys. Rev. A 74, 052322 (2006).

30. Albash, T., Boixo, S., Lidar, D. A. \& Zanardi, P. Quantum adiabatic markovian master equations. New J. Phys. 14, 123016 (2012).

\section{Acknowledgements}

MHA is grateful to A.J. Berkley, M.W. Johnson, R. Liu, T. Mahon, F. Nori, A. Yu. Smirnov, and $\mathrm{B}$. Wilson for discussions and comments.

\section{Author contributions}

All authors contributed equally to all aspects of this work.

\section{Additional information}

Supplementary information accompanies this paper at http://www.nature.com/ scientificreports

Competing financial interests: The authors declare no competing financial interests.

License: This work is licensed under a Creative Commons

Attribution-NonCommercial-NoDerivs 3.0 Unported License. To view a copy of this license, visit http://creativecommons.org/licenses/by-nc-nd/3.0/

How to cite this article: Deng, Q., Averin, D.V., Amin, M.H. \& Smith, P. Decoherence induced deformation of the ground state in adiabatic quantum computation. Sci. Rep. 3 1479; DOI:10.1038/srep01479 (2013). 